\title{
Double Stopband Bilayer Photonic Crystal Based Upconversion Fluorescence PSA Sensor
}

Songtao Hu ${ }^{\mathrm{a} 1}$, Hongwei Xu ${ }^{\mathrm{b} 1}$, Bingshuai Zhou ${ }^{\mathrm{a}}$, Shihan $\mathrm{Xu}^{\mathrm{c}}$, Bo Shen ${ }^{\mathrm{a}}$, Biao Dong ${ }^{\mathrm{a}}$, Ze Yin ${ }^{\mathrm{a}}$, Sai, $\mathrm{Xu}^{\mathrm{d}}$, Liheng Sun ${ }^{\mathrm{a}}$, Jiekai $\mathrm{Lv}^{\mathrm{a}}$, Jiahe Wang ${ }^{\mathrm{a}}$, Wen $\mathrm{Xu}^{\mathrm{a}}$, Xue Bai ${ }^{\mathrm{a}}$, Lin $\mathrm{Xu}^{\mathrm{a}}$, Svetlana Mintova ${ }^{\mathrm{e}}$, Hongwei Song ${ }^{\mathrm{a}^{*}}$

${ }^{a}$ State Key Laboratory on Integrated Optoelectronics, College of Electronic Science and Engineering, Jilin University, 2699 Qianjin Street, Changchun, 130012, P. R. China.

${ }^{b}$ Department of Mechanics and Engineering Science, College of Engineering, Peking University, Beijing, 100871, China.

${ }^{c}$ Department of Bioengineering, University of Washington, Seattle, United States.

${ }^{d}$ School of Science, Dalian Maritime University, Dalian 116026, People's Republic of China.

${ }^{e}$ Laboratoire Catalyse \& Spectrochimie, University of Caen, CNRS, 6, boulevard du Maréchal Juin, 14050 Caen Cedex, France

${ }^{1}$ These authors contributed equally to this work.

\section{Corresponding Author:}

*Email: dongb@jlu.edu.cn

*Email: songhw@jlu.edu.cn 


\begin{abstract}
Precise detection on tumor markers is significant for the early diagnosis on cancer. Upconversion fluorescence probes are ideal for tumor marker sensing, but still subject to bottleneck problems of low emission efficiency/strength. In this work, a novel bilayer of poly(methyl methacrylate) opal photonic crystal (PMMA OPC) with double photonic stop bands was fabricated to match the excitation field and emission field of the upconversion nanoparticles (UCNPs) at the same time, and greatly enhance their upconversion fluorescence intensity. Herein, $\mathrm{NaYF}_{4}: \mathrm{Yb}^{3+}, \mathrm{Er}^{3+} \mathrm{UCNPs}$ and $\mathrm{NaYF}_{4}: \mathrm{Yb}^{3+}, \mathrm{Er}^{3+} @ \mathrm{NaYF}_{4}: \mathrm{Yb}^{3+}, \mathrm{Nd}^{3+} \mathrm{UCNPs}$ with a core-shell structure were employed as a core, which could be excited by $980 \mathrm{~nm}$ and $808 \mathrm{~nm}$ light, respectively. With the optimized geometry structure, the upconversion emission intensity was enhanced over 150 folds under the excitation of both $808 \mathrm{~nm}$ and $980 \mathrm{~nm}$ laser. Through the $\mathrm{NaYF}_{4} / \mathrm{OPC}$ biochip and florescence resonant energy transfer (FRET) sensing strategy, the lowest detection limit of $0.01 \mathrm{ng} / \mathrm{mL}$ with good linear relationship was obtained ranging from $0.1 \mathrm{ng} / \mathrm{mL}$ to $10 \mathrm{ng} / \mathrm{mL}$. The power density of the pumping light was as low as $1.7 \mathrm{~W} / \mathrm{cm}^{2}$. This enhanced upconversion tumor marker biochip sensor provides a promising and powerful tool for facile early diagnosis on cancer markers.
\end{abstract}

\title{
Keywords
}

Upconversion fluorescence enhancement, double stop band, photonic crystal, tumor marker detection. 


\section{Introduction}

Cancer is one of the main diseases that endanger human life and health. Conventional detection methods, such as magnetic resonance imaging, and computed tomography, are mainly applied in clinical diagnosis with high sensitivity but restricted by complex equipment, high cost and time-consuming.[1-7] It's important to design convenient and efficient methods for early detection of tumors, especially for home-based self-screening. Note that the occurrence of cancer is closely associated with the abnormal expression of tumor markers, and the precise identification of potential cancer biomarkers in patient samples is a key factor in the early cancer diagnosis and an estimation of the cancer development stage.[8-12]

Recent years, different kinds of innovative tumor marker detection methods have been developed. Among them, biochip-based device for biomarker detection is an important assay not only in clinical diagnostic fields, but also in home-based self-screening, due to the portability, flexibility, and short processing time on samples. In addition, new technological progress in the biomarker analysis occurs with recent innovations on optical detection methods with fluorescence readout.[13, 14] Unlike traditional luminescent materials, such as quantum dots and dyes,[15-18] rare earth doped upconversion nanoparticles (UCNPs) are able to transform NIR light into visible light,[19-26] with less damage on biospecimen, meanwhile, avoids background fluorescence during detection in comparison with conventional fluorescence probes.[27-31] Furthermore, UCNPs can be applied as a suitable energy donor in fluorescence resonant energy transfer (FRET) for the detection on tumor markers, and lots of biochip-based diagnostics of cancer for point-of-care (POC) applications were developed. $[13,14]$

The goal of the POC diagnosis is to detect biomarkers with the requisite sensitivity and accuracy to meet the needs of clinical diagnosis and home-based self-screening. However, low fluorescence quantum yield of UCNPs probe limits their clinical applications on biochip devices for tumor marker analysis. Nowadays, enhancing the intensity of upconversion fluorescence is a challenge to expand their biological applications. To regulate upconversion florescence, core-shell structure and 
local field effect became the main means to enhance the intensity of upconversion fluorescence. Normally, the fluorescence intensity can be improved[32-36] by preparing passivation shell, which, however, may cause the increasing size of UCNPs. In biological detection, small size NPs with large specific surface area are preferred for the enough active sensing site, and meanwhile, low power density of the excitation light is applied on specimens,[37] both of which limit the emission intensity of UCNPs.[38, 39] To enhance the emission intensity, various methods based on local field effect are developed, including photonic crystal effect[40-42] and surface plasma effect[43-45]. They have been proved as effective ways to enhance fluorescence intensity, especially for small size NPs and with a low excitation power. For upconversion based biochips, local field effect with the advantage on the fluorescence enhancement, might greatly improve the detection performance and satisfy the clinical requirements for patient sample assays.

Since the discovery of the photonic crystal effect, it has received extensive attentions on optical regulation, especially for the intensity enhancement excited by the low-power light. Opal photonic crystal (OPC) with photonic stop bands can prevent or scatter the light that falls into the band gaps.[46] Once photonic stop band matches with the excitation light or emission light wavelength, upconversion emission intensity of UCNPs could increase over dozens of folds, [47] with putting UCNPs on the surface of OPCs. In our previous work, through combining PMMA OPC effect and surface plasma effect, the enhancement factor of upconversion emission of $\mathrm{NaYF}_{4}: \mathrm{Yb}^{3+}, \mathrm{Er}^{3+} \mathrm{NPs}$ reached 1000 -fold[48]. The photonic crystal stop band coupling with the upconversion excitation field has been proved to greatly increase the upconversion fluorescence intensity. Until now, synchronous coupling the upconversion excitation and emission fields has not been reported yet.

In this work, in order to promote the potential clinical utility of upconversion based biochip devices, a novel bilayer PMMA OPC with double photonic stop band was designed. In this biochip, based on the previous research results, bilayer photonic crystal structure was used to regulate the stop band to match the excitation field and emission field of the upconversion fluorescence, respectively. Because 
$\mathrm{NaYF}_{4}: \mathrm{Yb}^{3+}, \mathrm{Er}^{3+}$ and core-shell structure $\mathrm{NaYF}_{4}: \mathrm{Yb}^{3+}, \mathrm{Er}^{3+} @ \mathrm{NaYF}_{4}: \mathrm{Yb}^{3+}, \mathrm{Nd}^{3+}$ UCNPs were chose for the energy donor, which can be excited by $980 \mathrm{~nm}$ and $808 \mathrm{~nm}$ light, respectively. The double stop bands were regulated to $980 \mathrm{~nm}$ and $808 \mathrm{~nm}$ (excitation) and $545 \mathrm{~nm}$ (emission) band of UCNPs respectively (Fig. 1). Through the $\mathrm{NaYF}_{4} / \mathrm{OPC}$ hybrid structure, the upconversion emission intensity was enhanced over 150 folds under excitation of both $808 \mathrm{~nm}$ and $980 \mathrm{~nm}$, which was superior to the conventional monolayer OPC. The geometry of the dual photonic crystal was also investigated. Utilizing the optimized structure, an upconversion fluorescence sensor was fabricated to detect a tumor marker, prostate specific antigen (PSA). The detection limit of $0.01 \mathrm{ng} / \mathrm{mL}$ with good linear relationship in the range of $0.1 \sim 10$ $\mathrm{ng} / \mathrm{mL}$ was obtained under the excitation power density of $1.7 \mathrm{~W} / \mathrm{cm}^{2}$. Since the upconversion fluorescence is greatly enhanced, in the same condition, the excitation power density can be lowered with minimal damage on the sample. The remarkable performance of our PSA-detection biochip could lead to further development in clinical diagnostic applications, as well as in home-based self-screening.

(a)

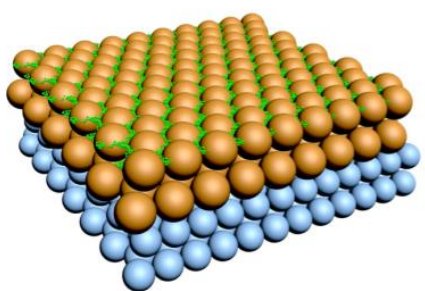

$980 / 808 \mathrm{~nm}$ OPC (b) $\quad 980 / 808 \mathrm{~nm}$ Laser

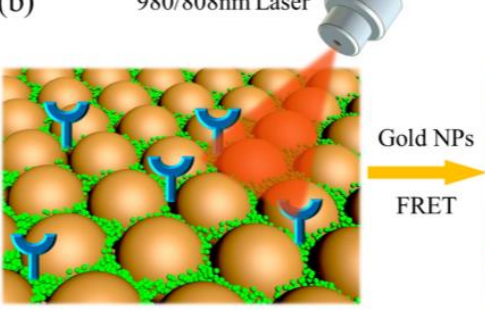

(c) $\quad 980 / 808 \mathrm{~nm}$ Laser

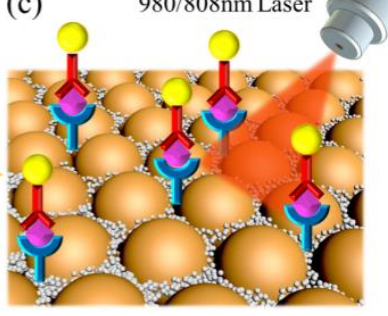

Anti-PSA1 YAnti-PSA2

Fig. 1 Schematic illustration of detection system. (a) UCNPs/bilayer OPC hybrid fluorescence film. (b) Fluorescence film modified by poly-dopamine film and capture anti-PSA, excited by 980/808 nm laser. (c) After connecting with PSA and detection anti-PSA modified gold nanoparticles, emission light intensity of UCNPs quenches.

\section{Experimental}

Preparation of double stop band OPC: PMMA OPC consisted of PMMA microsphere was prepared through a MMA polymerization process. Clean glass substrate was put into PMMA colloidal suspension (5\% solid content) vertically and placed in $35^{\circ} \mathrm{C}$ for $24 \mathrm{~h}$, the PMMA microsphere would be assembled orderly on glass 
substrate by surface tension along with the evaporation process. After this, OPC was put into $120^{\circ} \mathrm{C}$ for $1 \mathrm{~h}$ to enhance its physical strength. The position of PSB could be controlled by changing the size of PMMA microsphere. In this work, three kinds of OPC with PSB at $545 \mathrm{~nm}, 808 \mathrm{~nm}$, and $980 \mathrm{~nm}$ were prepared. Next, we fixed OPC in beaker vertically and injected deionized water into beaker slowly by micro syringe pump with rate of $10 \mathrm{~mL} / \mathrm{h}$, OPC film on glass substrate would be peeled by surface tension. After OPC was peeled, we used another OPC to fish it, and strengthened it in $60^{\circ} \mathrm{C}$ for $2 \mathrm{~h}$.

Synthesis of $\mathrm{NaYF}_{4}: \mathbf{Y b}^{3+}, \mathbf{E r}^{3+}$ core nanocrystals: $0.78 \mathrm{mmol} \mathrm{YCl}_{3}, 0.20 \mathrm{mmol}$

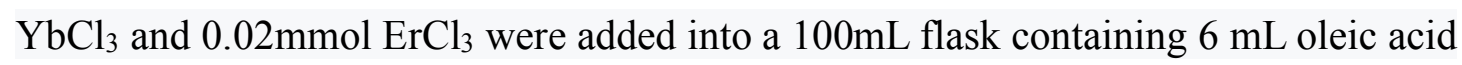
and $15 \mathrm{ml} \mathrm{1-octadecene.} \mathrm{The} \mathrm{mixture} \mathrm{was} \mathrm{stirred} \mathrm{at} 160^{\circ} \mathrm{C}$ for $60 \mathrm{~min}$ to form the lanthanide-oleate complexes and then cooled down to $40^{\circ} \mathrm{C}$. Next, dropwise adding 6 $\mathrm{mL}$ of methanol solution containing $4 \mathrm{mmol} \mathrm{NH}_{4} \mathrm{~F}$ and $2 \mathrm{mmol} \mathrm{NaOH}$ into flask and the resultant solution was gently stirred for $30 \mathrm{~min}$. After fully stirred, the solution was heated to $120^{\circ} \mathrm{C}$ to remove methanol. Finally, the solution was further heated to $300^{\circ} \mathrm{C}$ for 90 min to obtain $\mathrm{NaYF}_{4}: \mathrm{Yb}^{3+}, \mathrm{Er}^{3+}$ nanoparticles. The whole preparation took place with the nitrogen protection. Then the solution was cooled down to room temperature. The resulting NPs were collected by centrifugation in ethanol at 9,500 rpm for $15 \mathrm{~min}$, washed with absolute ethanol-cyclohexane (1: $3 \mathrm{v} / \mathrm{v})$ two times and dispersed in cyclohexane for the following experiments.

\section{Synthesis of $\mathrm{NaYF}_{4}: \mathrm{Yb}^{3+}, \mathrm{Er}^{3+} @ \mathrm{NaYF}_{4}: \mathrm{Yb}^{3+}, \mathrm{Nd}^{3+}$ core-shell nanocrystals:}

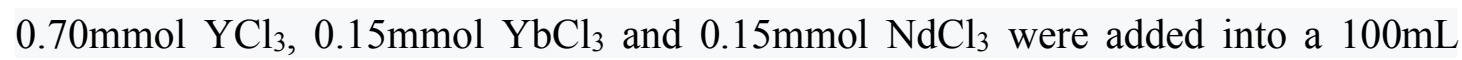
flask containing $6 \mathrm{~mL}$ oleic acid and $15 \mathrm{ml} \mathrm{1-octadecene.} \mathrm{The} \mathrm{mixture} \mathrm{was} \mathrm{stirred} \mathrm{at}$ $160^{\circ} \mathrm{C}$ for $60 \mathrm{~min}$ to form the lanthanide-oleate complexes and then cooled down to $40^{\circ} \mathrm{C}$. Next, $6 \mathrm{~mL}$ cyclohexane solution was added to coat $1 \mathrm{mmol} \mathrm{NaYF} 4: \mathrm{Yb}^{3+}, \mathrm{Er}^{3+}$ NPs. After fully stirred for $30 \mathrm{~min}$, the solution was heated to $90^{\circ} \mathrm{C}$ to remove methanol cyclohexane and then cooled down to $40^{\circ} \mathrm{C}$. Next steps are the same with the last part.

Preparation of gold nanoparticles: Gold NPs were prepared through sodium citrate reduction method. Firstly, $50 \mathrm{~mL} \mathrm{HAuCl}_{4}$ solution $(1 \mathrm{mM})$ was added into flask, 
the solution was stirred and heated in oil bath to boiling, then rapidly added sodium citrate solution $(39 \mathrm{mM})$. The whole progress required condensation reflux. After a few minutes, the solution turned to wine red. Being heated $15 \mathrm{~min}$ continually, and then the solution was cooled down to room temperature. Gold NPs prepared through sodium citrate reduction method could be modified with antibody through electrostatic adsorption. Take $1 \mathrm{~mL}$ gold NPs solution, collected by centrifugation at $12,000 \mathrm{rpm}$ for $15 \mathrm{~min}$ and re-dispersed in $1 \mathrm{~mL}$ deionized water. Then add $60 \mu \mathrm{L} 0.1$ $\mathrm{mg} / \mathrm{mL}$ detection anti-PSA. After shaking for $1 \mathrm{~h}$ and storing at $4^{\circ} \mathrm{C}$ for $10 \mathrm{~h}$, detection anti-PSA modified gold NPs were collected by centrifugation at $12,000 \mathrm{rpm}$ for $15 \mathrm{~min}$ again.

Fabrication of PDA/NaYF $/ \mathrm{OPC}_{4}$ hybrid film: Glass and OPC films were vertically dropped into $\mathrm{NaYF}_{4}$ cyclohexane solution $(0.1 \mathrm{mg} / \mathrm{mL})$ and placed at $28^{\circ} \mathrm{C}$ for 6h. With the volatilization of cyclohexane, the $\mathrm{NaYF}_{4}$ UCNPs will self-assemble onto the surface of glass and OPC films. Vertically putting hybrid films into dopamine TRIS solution $(0.6 \mathrm{mg} / \mathrm{mL}, \mathrm{pH}=8.0)$ for $1 \mathrm{~h}$ and let dopamine self-polymerization at the surface of hybrid films for binding antibody.

Detection Section: PSA、capture antigen and detection antigen were purchased from Shanghai Linc-Bio science company(Basic information in Supplementary Material). The capture anti-PSA $(0.1 \mathrm{mg} / \mathrm{mL})$ was modified onto the surface of hybrid film. Next, the samples with different concentration of PSA were added onto the surface of hybrid film sensor, after incubation for 10 minutes, detection area was washed adequately by PBS buffer, then BSA blocking buffer and gold NPs modified with detection anti-PSA were orderly added and modified onto the surface of hybrid film sensor. It also needed 10 minutes for incubation in each step. Finally, detection area was washed by PBS buffer again. In next step, the emission spectra of detection area were recorded with fluorescence spectrometer equipped with 980 and $808 \mathrm{~nm}$ excitation lasers. For the tumor marker detection with confocal microscope, we just chose one detection area and added excessive capture anti-PSA, and then orderly added PSA sample and detection anti-PSA modified GNPs for multi times. The 
amount of capture anti-PSA was calculated to be enough to meet the requirement of six experiments. $0,0.01,0.1,2,5,12.5 \mathrm{ng} / \mathrm{mL}$ PSA was added to the same detection area and the GNPs with same amount was added after each PSA sample addition. The real time tumor marker detection images in the sensing area and the corresponding spectra were recorded and scanned with confocal microscope.
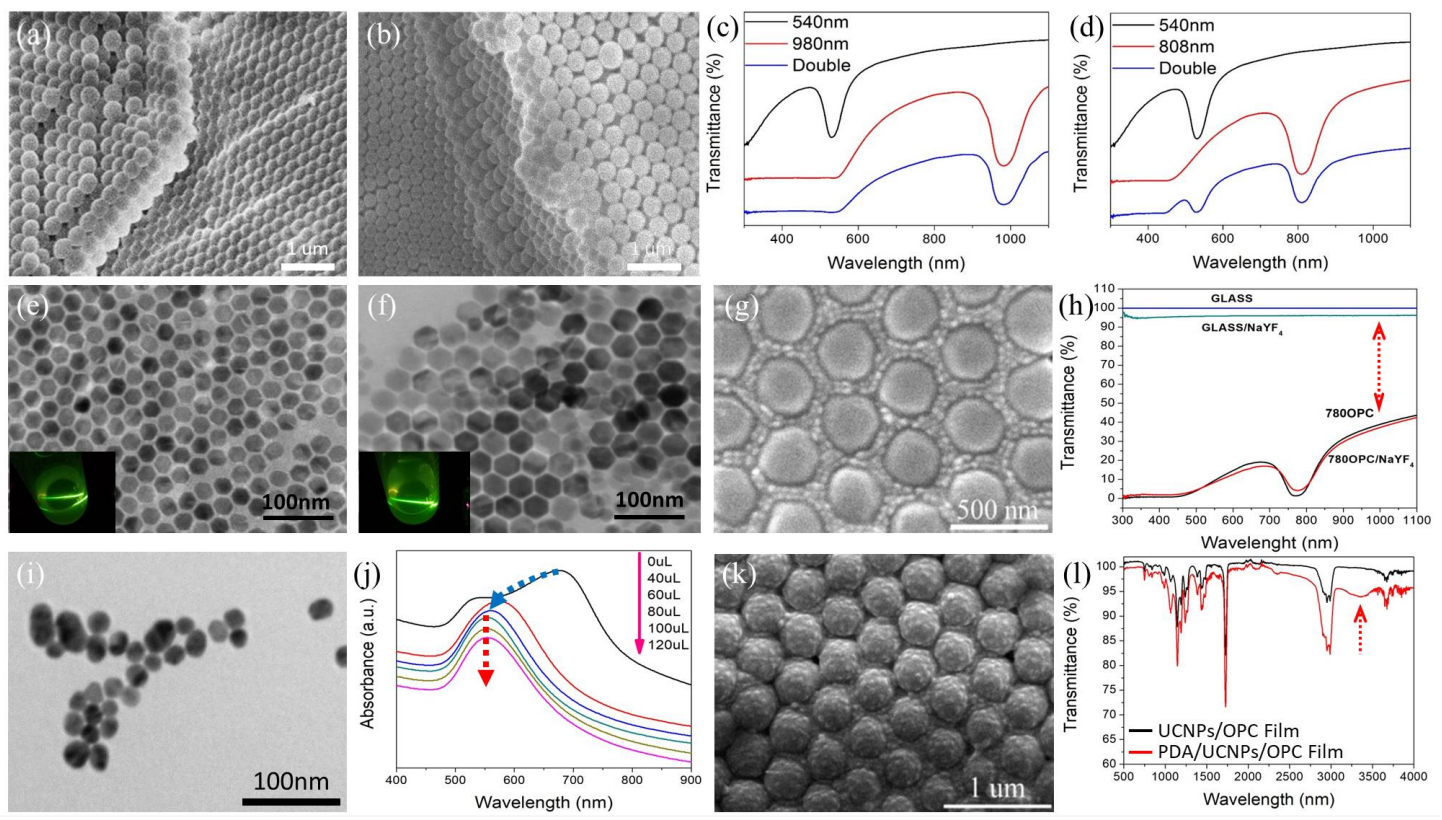

Fig. 2 (a-d) SEM image and transmitted spectra of 980/545 nm and 808/545 nm double stop band OPC. (e-f) TEM image of UCNPs $\mathrm{NaYF}_{4}: \mathrm{Yb}^{3+}, \mathrm{Er}^{3+}$ and $\mathrm{NaYF}_{4}: \mathrm{Yb}^{3+}, \mathrm{Er}^{3+} @ \mathrm{NaYF}_{4}: \mathrm{Yb}^{3+}, \mathrm{Nd}^{3+}$. (g) SEM image of UCNPs/OPC hybrid film. (h) Transmitted spectra of glass substrate, UCNPs/glass, OPC film, and UCNPs/OPC hybrid film. (i) TEM image of gold nanoparticles. (j) Absorption spectra of gold nanoparticles, they are modified by different amount of detection antibody and then adding $\mathrm{NaCl}$ solution. (k) SEM image of PDA/UCNPs/OPC hybrid film. (l) Fourier infrared spectra of PDA/UCNPs/OPC hybrid film. (l) FTIR spectra of poly-dopamine modified fluorescence film.

\section{Results and discussions}

PSA is an important tumor marker of prostatic cancer, and the value of healthy person is $4 \mathrm{ng} / \mathrm{mL}$. Above this value, the risk of tumor increases, and accurate detection of this tumor marker is important for early screening of prostatic cancer.

Figure 1 shows the design of bilayer upconvesion sensor and the principle of PSA detection. Through antibody-antigen-antibody combination, UCNP fluorescence donor can be linked with gold nanoparticles (NPs) receptor, and then the concentration of antigen can be quantified through measuring the decreased emission 
intensity due to the fluorescence quenching. After modifying the UCNPs on the surface of the photonic crystal, poly-dopamine (PDA) was further modified on the surface of UCNPs film for connecting with capture anti-PSA. Next, gold NPs with absorption peak at $560 \mathrm{~nm}$ was prepared, which matched with green emission peak of $\mathrm{Er}^{3+}$, and the anti-PSA was further modified on gold NPs by electrostatic adsorption for detection.[49-51] In this system, we detected the concentration of PSA in PBS buffer solution under both $980 \mathrm{~nm}$ and $808 \mathrm{~nm}$ excitation. In the process, dropping capture anti-PSA, sample, BSA blocking buffer, and gold nanoparticles modified with detection anti-PSA onto fluorescence film in order, then testing the emission spectra of the detection area. BSA blocking buffer plays the function of anti-fouling to prevent the nonspecific adsorption of detection anti-PSA. Both capture anti-PSA and detection anti-PSA can target the PSA to form a sandwich structure that shortens the distance between the UCNPs and the gold NPs. Since the green emission from UCNPs and the plasma absorption of gold NPs overlap, efficient energy transfer can happen. The emission intensity of $545 \mathrm{~nm}$ light would reduce as the concentration of PSA increases.

Fig. 2a-d shows the SEM images and transmittance spectra of $980 / 545 \mathrm{~nm}$ and $808 / 545 \mathrm{~nm}$ double stop band OPC, respectively. The bilayer structure of OPC is composed of PMMA spheres with two different sizes. From the SEM images, it can be observed that PMMA spheres are uniform and regular in arrangement, and both of the layers maintain good integrity. In Fig. 2a and b, the photonic crystals with band gaps around 980 and 808 are both in the upper layer, and the photonic crystals with band gaps around 545 are at down layer. It should be mentioned that, through the photonic crystal grafting method mentioned in experiment section, the photonic crystals of different sizes can be geometrically arranged separately, and this method is also applicable for flexible substrate, such as polyethylene glycol terephthalate (PET) substrate.[48] The bilayer photonic crystal films can match both the excitation field and the emission field together, which is important for studying the interaction between the excitation field and the emission field with the photonic band gap, and exploring the optimal fluorescence enhancement mode. From transmittance spectra, 
two photonic stop bands matching the excitation light and emission light, can be observed and the position of photonic stop band could be regulated by adjusting the size of PMMA spheres. The black and red lines correspond to the transmittance spectra of a single-layer photonic crystal, and the stop band position corresponds to the size of the PMMA. When the two photonic crystals are combined, transmission spectra (blue line) with double stop bands are obtained, which further suggests the successful preparation of double stop band bilayer photonic crystal structure. It is worth mentioning that, in the 980/545 nm bilayer photonic crystal structure, although the stop band of $545 \mathrm{~nm}$ is relatively shallow, it still provides contribution to the fluorescence enhancement.

From the TEM images of UCNPs (Fig. 2e, f), $\mathrm{NaYF}_{4}: \mathrm{Yb}^{3+}, \mathrm{Er}^{3+} \mathrm{UCNPs}$ are 23 nm, and the $\mathrm{NaYF}_{4}: \mathrm{Yb}^{3+}, \mathrm{Er}^{3+} @ \mathrm{NaYF}_{4}: \mathrm{Yb}^{3+}, \mathrm{Nd}^{3+} \mathrm{UCNPs}$ are $34 \mathrm{~nm}$, both of which have good morphology of hexagonal, uniform sizes and good dispersion. The size of UCNPs can be tuned by changing the reaction temperature from $280^{\circ} \mathrm{C}$ to $320^{\circ} \mathrm{C}$ and the size of UCNPs increases with the increasing reaction temperature. The size of $\mathrm{NaYF}_{4}: \mathrm{Yb}^{3+}, \mathrm{Er}^{3+} @ \mathrm{NaYF}_{4}: \mathrm{Yb}^{3+}, \mathrm{Nd}^{3+} \mathrm{UCNPs}$ increased, due to the modification of the shell, and the thickness of the shell was about $5 \mathrm{~nm}$. Both UCNPs can emit strong green light under the excitation of 808 and $980 \mathrm{~nm}$ lights. The $980 \mathrm{~nm}$ excitation can generate upconversion emission of $\mathrm{Er}^{3+}$ by the two-step sensitization of $\mathrm{Yb}^{3+}$ ions. The emission process under $808 \mathrm{~nm}$ excitation is more complicated, in which the $\mathrm{Nd}$ ions in the shell absorbs the $808 \mathrm{~nm}$ photon and then transfers energy to nearby $\mathrm{Yb}^{3+}$. Through the core-shell structure, this energy can be effectively transferred to the $\mathrm{Yb}^{3+}$ ions in the core and this migrated energy is finally transferred to the Er ions by two-step energy transfer, as shown in the schematic diagram. (Fig. 3)

The SEM image of the UCNPs/OPC hybrid films (Fig. 2g) shows that uniform distribution of UCNPs in the crevices between PMMA spheres. When the position of stop band matches with excitation wavelength, the excitation field can be amplified, and even with low-power excitation, upconversion fluorescence will be enhanced. Especially when UCNPs are located in the interstices between PMMA spheres, the luminescent intensity of UCNPs in those interstices could be further enhanced. The 
PMMA photonic crystal prepared by the self-assembly method is very sensitive to water, and water can damage the photonic crystal structure. In this process, the surface of the photonic crystal is modified with a thin hydrophobic layer, which can protect the photonic crystal structure when detecting aqueous solutions. In order to verify the role of the hydrophobic protective layer, we tested the transmittance spectra of UCNPs/bilayer OPC hybrid film before and after placed in water for 4 hours. Benefit from hydrophobic oleic acid ligands on the surface of UCNPs (Fig. S1), UCNPs/bilayer OPC hybrid film shows good structural integrity after placed in water and the transmittance spectra of bilayer OPC base keeps intact, demonstrating the well water stability of UCNPs/bilayer OPC hybrid film. After modified with poly-dopamine, SEM image and Fourier Transform Infrared Spectroscopy (FTIR) spectra of hybrid film was tested (Fig. 2k, 1). Contrast with Fig. 2g, a dense film in SEM image could be observed. In FTIR spectra, the new absorption peak between $3100 \mathrm{~nm}$ and $3500 \mathrm{~nm}$ is assigned to the $\mathrm{N}-\mathrm{H}$ bond from poly-dopamine, which can be further used to connect with capture anti-PSA.

When studying the photonic crystal effect on the upconversion fluorescence enhancement, it is necessary to determine that the amount of UCNPs on the photonic crystal and the glass substrate are equal, so it is meaningful to compare the upconversion fluorescence enhancement. In the experimental process, same UCNPs solution were modified on the surface of photonic crystal and glass substrate at the same time, to ensure the comparability of their optical properties. In addition, we tested transmittance spectroscopy of glass substrate, UCNPs/glass, OPC film, and UCNPs/OPC hybrid film as shown in Fig. 2h. In the calculations of enhancement factor, it is necessary to avoid the influence of PSB, so the OPC with PSB of $780 \mathrm{~nm}$ is chosen and the transmittance at $980 \mathrm{~nm}$ is tested. Through Beer-Lambert law $\left(E_{i}=\log \left(1 / T_{i}\right), i=1,2\right)$, the values of extinction were calculated $\left(E_{1}=1.79\right.$ and $\left.E_{2}=1.97\right)$. This result indicates that the amount of UCNPs on glass substrate and OPC film are quite close. Thus the influence of the amount of UCNPs on different substrates can be neglected on calculation of the upconversion enhancement factors.

As the energy acceptor, gold NPs in Fig. 2i show uniform size and good 
dispersion. The absorption peak locates at $560 \mathrm{~nm}$, which overlaps with the green emission of $\mathrm{Er}^{3+}$ (Fig. S2a). To confirm the appropriate concentration of gold NPs, the concentration of prepared gold NPs was adjusted to be $0.002,0.02,0.2,0.6$, $1.0 \mathrm{mg} / \mathrm{mL}$, then $4 \mathrm{uL}$ PBS and five kinds of adjusted gold NPs were respectively added onto fluorescence film and the emission spectrum of every detection area was tested. In our detection system, when the concentration of gold NPs progress was between $0.02 \mathrm{mg} / \mathrm{mL}$ to $0.2 \mathrm{mg} / \mathrm{mL}$, most of emission light was quenched and the FRET progress reached saturation point (Fig. S2b, c). So gold NPs with concentration of $0.2 \mathrm{mg} / \mathrm{mL}$ was chose to process subsequent experiment. To demonstrate that the detection anti-PSA was connected with gold NPs, six controlled trials were conducted. Firstly, $0,40 \mu \mathrm{l}, 60 \mu \mathrm{L}, 80 \mu \mathrm{L}, 100 \mu \mathrm{L}$, and $120 \mu \mathrm{L} 0.1 \mathrm{mg} / \mathrm{mL}$ detection anti-PSA were added into $1 \mathrm{~mL}$ prepared gold NPs solution, respectively. After uniformly oscillating for $1 \mathrm{~h}$, gold NPs were centrifuged and re-dispersed in $1 \mathrm{~mL}$ deionized water. Finally adding $100 \mu \mathrm{L} 1 \mathrm{mg} / \mathrm{mL} \mathrm{NaCl}$ solution in each sample and test their absorption spectra (Fig. 2j). After mixed with $\mathrm{NaCl}$ solution, the absorption spectra of pure gold NPs solution were broadened obviously due to the agglomeration of gold nanoparticles. More antibodies were connected with gold NPs as their concentration increased, which further weakened the agglomeration of gold NPs, so that the absorption spectra broaden disappeared gradually. This absorption trend indicates that the antibody was successfully modified on the surface of gold NPs. 

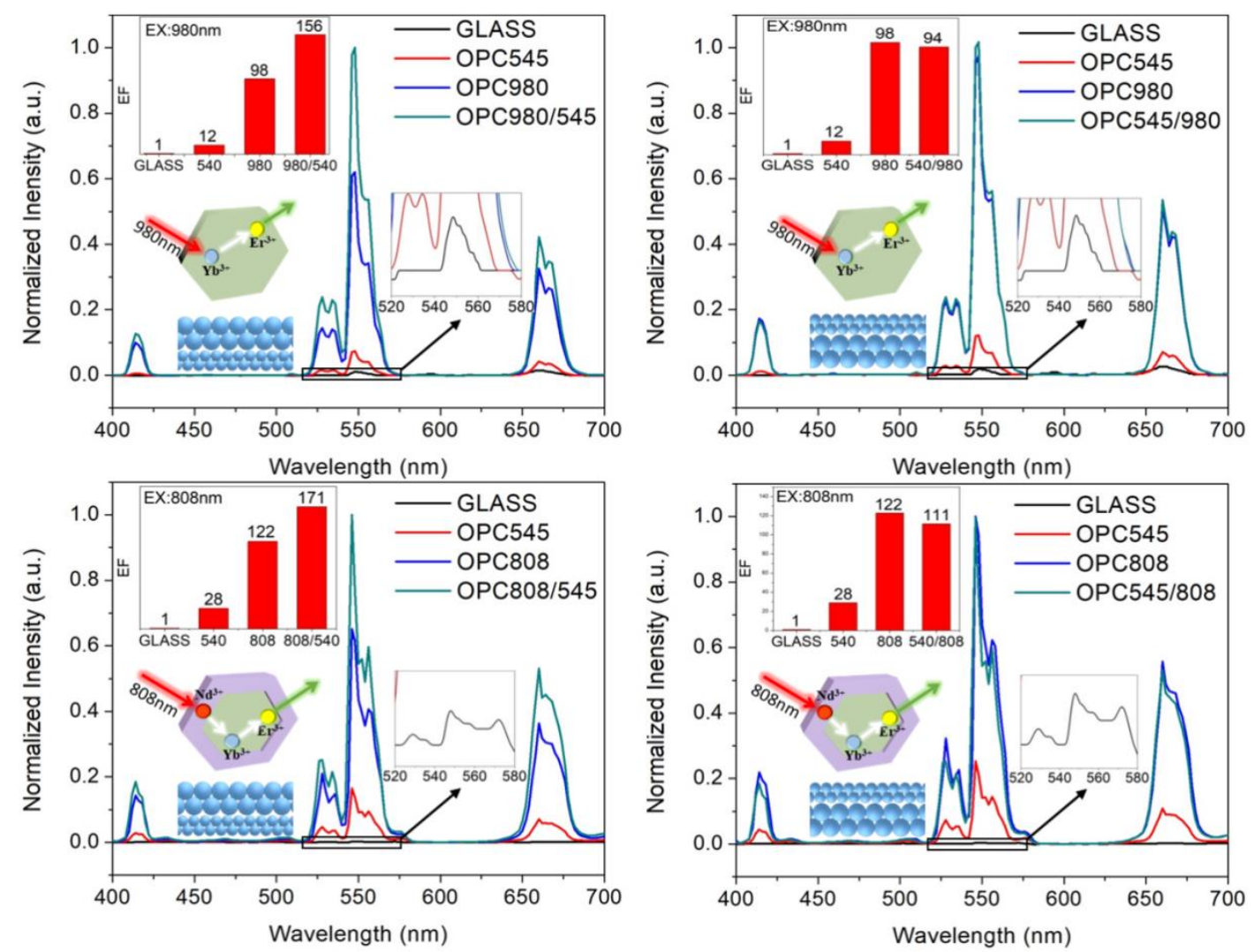

Fig. 3 Emission spectra, enhancement factory and schematic diagram of energy transfer progress of four kinds of fluorescence films. (a)UCNPs/980 nm OPC/545 nm OPC (b)UCNPs/545 nm OPC/980 nm OPC (c)UCNPs/808 nm OPC/545 nm OPC (d)UCNPs/545 nm OPC/808 nm OPC.

In our previous work,[44, 48, 52] remarkable enhancement on the intensity of upconversion fluorescence was realized in the coupling of the photonic crystal stop band with the upconversion excitation field. Upconversion fluorescence is a multiphoton process, therefore the fluorescence efficiency is relatively low. In biological detection, it is important to increase the fluorescence intensity. In this work, we use the local field effect of PMMA OPC to increase the upconversion fluorescence intensity. The band gap of the photonic crystal structure can be adjusted by the size of the PMMA sphere. When the photonic stop band is consistent with the wavelength of the incident light, the light will scatter many times on the surface of OPC, especially in the crevices between PMMA spheres. Since UCNPs are placed in the crevices of photonic crystal microspheres, when the excitation light intensity on the surface of OPC is greatly enhanced, then the up-conversion fluorescence is greatly enhanced. According to this principle, the emission light of UCNPs can also be enhanced, as 
long as the position of the upconversion emission light and the photonic crystal stop band overlaps. Therefore, we have designed double-layer photonic crystal structure, so that their stop bands can be respectively coupled with the excitation light and emission light of UCNPs, causing that the excitation field and emission field of UCNPs can be simultaneously enhanced, and the intensity of up-converted fluorescence can be further improved. We found that when the photonic stop band is coupled with the excitation field, the better fluorescence enhancement can be obtained, so the upper photonic crystal stop band is set at 980 and $808 \mathrm{~nm}$, and the down layer is at $545 \mathrm{~nm}$ couping with emission field, to obtain the best upconversion fluorescence. As for the important factors related to fluorescence enhancement, it should be the position of the photonic band gap and the power of the excitation light. The position of the photonic band gap is controlled by the size of the PMMA microsphere. Once the optical band gap position mismatches with excitation light or emission light wavelength, the enhancement factor will decrease rapidly. Regarding excitation power and enhancement factor, we found that when the lower excitation power density is employed, greater enhancement factor can be obtained. In addition, the size of fluorescence nanoparticles also influences the enhancement factor. Bigger size of nanoparticles results in lower enhancement factor. Although clear quantitative relation is hard to get, we can build a more appropriate enhancement system for detection according to the variation trend. Normally, low power density of excitation light is meaningful and necessary for biological detection, which is needed to avoid damage to biological samples. Interestingly, our test system can obtain higher fluorescence enhancement when low power is used, showing that this system is suitable for biological detection which can protect biological samples and improve detection accuracy. In this work, we further introduced a bilayer photonic crystal and synchronously coupled the excitation and emission fields to enhance upconversion fluorescence. The work of synchronizing to couple the upconversion excitation and emission fields has not been reported yet. The difficulty lies in not only the fabrication, but also the spatial configuration and regularity, which are still unclear. The photonic crystal effect has a certain working distance, and UCNPs can obtain the maximum 
enhancement on the surface of the photonic crystal. With this bilayer photonic crystal, it is necessary to study the spatial order structure. In this work, as mentioned above, different spatial order of photonic crystals is designed to optimize the enhancement of upconversion emission. Fig. 3a-d shows emission spectra of two kinds of UCNPs excited by $980 \mathrm{~nm}$ and $808 \mathrm{~nm}$ laser, tested by spectrograph. Emission spectra and enhancement factor of UCNPs films on glass substrate, $545 \mathrm{~nm}$ OPC, 980/808 nm OPC, and double stop band OPC was compared. From spectra, compared with glass substrate, $545 \mathrm{~nm}$ emission intensity increased 12, 98, 156 folds under $980 \mathrm{~nm}$ excitation and 28, 122, 171 folds under $808 \mathrm{~nm}$ excitation. Light of corresponding wavelength was prevented by photonic stop band and was scattered on the surface of OPC, especially in the crevices between PMMA spheres. Obviously this effect was more effective to excitation light than emission light, and double stop band OPC showed much stronger enhancement performance than monolayer OPC. To confirm the influence of the spatial sequence of bilayer OPC with PSB of $545 \mathrm{~nm}$ and 980/808 $\mathrm{nm}$, controlled trial was conducted. From emission spectra, the trend of fluorescence enhancement of $980 \mathrm{~nm}$ part and $808 \mathrm{~nm}$ part is the same. When the $545 \mathrm{~nm} \mathrm{OPC}$ located on the upper side and 980/808 nm OPC on the down side, the enhancement factor was the same level with single 980/808 nm OPC. When the 980/808 nm OPC was on the upper side and the $545 \mathrm{~nm}$ OPC was on the down side, the enhancement factor was much higher than another sample. To get available light signal on glass substrate, these spectra were tested under a relatively high excitation light of 5 $\mathrm{W} / \mathrm{cm}^{-2}$. 

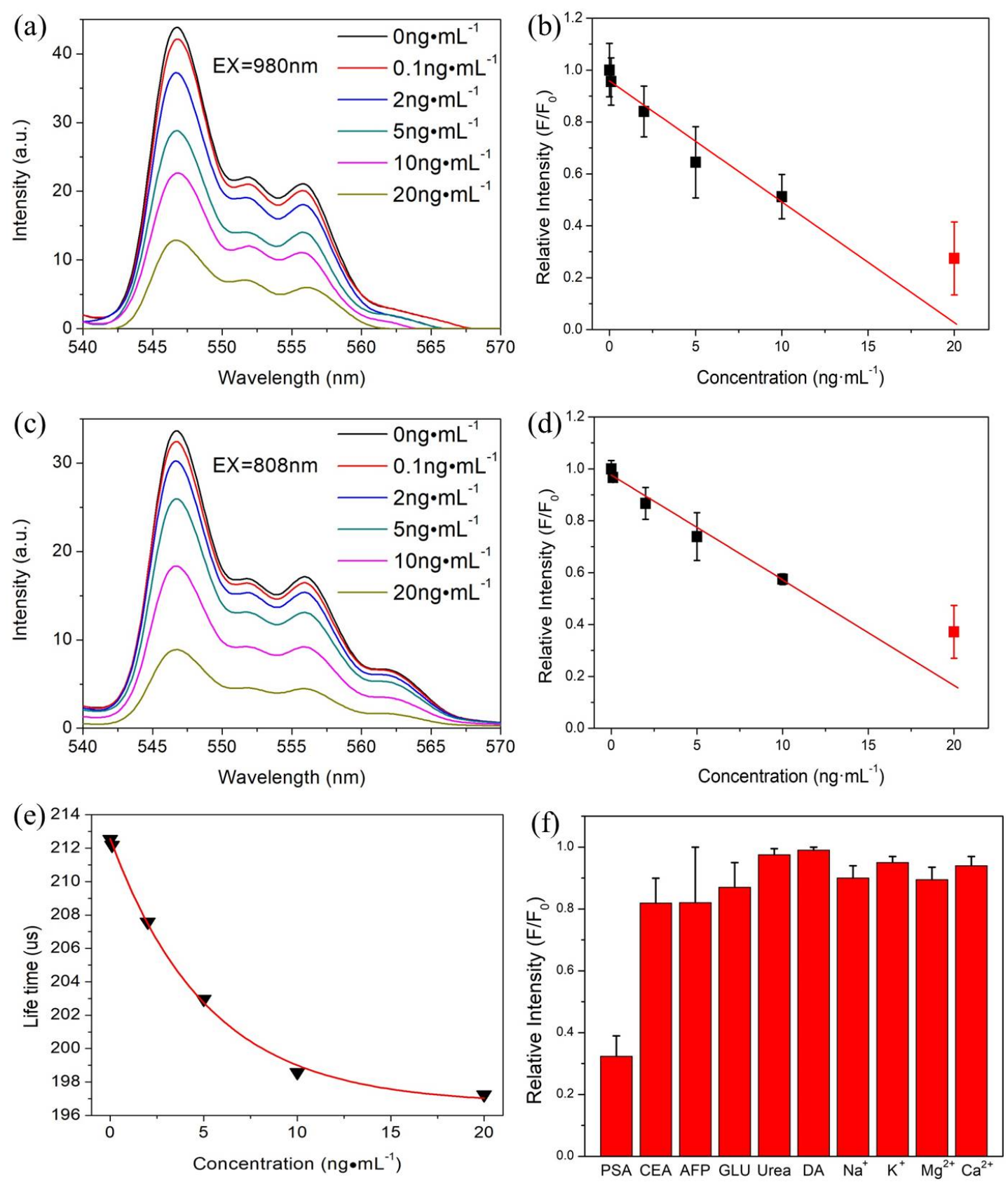

Fig. 4 (a-e) upconversion emission spectra of detection area, linear fitting and fluorescence lifetime result under $980 \mathrm{~nm}$ and $808 \mathrm{~nm}$ excitation in antigen detection. (f) specificity test result of PSA and other common substance in the blood.

Fig. 4 shows the PSA detection results tested by spectrograph. We chose $545 \mathrm{~nm}$ emission peak of $\mathrm{Er}^{3+}$ as detection position, and the emission intensity decreases as the increase of the concentration of PSA in sample (Fig. 4a, c). The correlation between emission intensity and PSA concentration was also calculated (Fig. 4b, d), which showed satisfactory linear relationship within the range of $0.1 \sim 10 \mathrm{ng} / \mathrm{mL}$ and 
the limit of detection (LOD) is $0.01 \mathrm{ng} / \mathrm{mL}$. When sample concentration is $20 \mathrm{ng} / \mathrm{mL}$, signal response showed a saturation trend and deviated from the fitting curve. Note that the PSA content of normal people is below $4 \mathrm{ng} / \mathrm{mL}$, and if PSA concentration is above $10 \mathrm{ng} / \mathrm{mL}$, risk of prostate cancer will significantly increase, our detection system meets the requirement of clinical detection well. Fig. S3 shows the detection result in PBS and blood sample. To test blood sample with low PSA concentration, blood sample with PSA concentration of about $2 \mathrm{ng} / \mathrm{mL}$ was diluted to $1 / 10$ of its original concentration and blood sample with PSA concentration of 0.2, 2.2, 5.2, 10.2, 20.2ng/mL was prepared, respectively. Compared with PBS solution, detection result of blood sample also shows very good linear relationship with a little slop change in the range of $0 \sim 10 \mathrm{ng} / \mathrm{mL}$, indicating that the detection system is promising for PSA detection in blood sample. When sample concentration is $20 \mathrm{ng} / \mathrm{mL}$, signal response showed a saturation trend and deviated from the fitting curve, which was consistent with previous result (Fig. 4b, d). The signal response of blood sample was slightly higher than that in PBS solution, which can be attributed to that, in our detection system, antigen-antibody reaction in blood sample might be impeded by viscosity of blood. Compared with PBS solution sample at the same concentration, less PSA in blood sample can be attached with capture antibody due to the viscosity of blood, so that less fluorescence quenching occurred in detection, which resulted in the difference in signal response. These results were tested under excitation light of 1.7 $\mathrm{W} / \mathrm{cm}^{-2}$. It should be mentioned that, the same amount of GNPs were added to each test, while excessive GNPs did not affect the results of the assay. The upconversion fluorescence intensity is only related to the amount of PSA, indicating that this sandwich structure is effective for narrowing the distance between the donor and the acceptor, and that other kind of contacts do not lead to energy transfer. Fig. 4e shows the fluorescence lifetime in each detection area. The lifetime decreases as the increase of the concentration of PSA in sample. However, compared with emission intensity, the descend range of lifetime is much smaller. The reason may be that under present testing condition, emission spectra could be tested in a very low excitation power, so only surface layer UCNPs were excited and quenched by gold nanoparticles. However, 
the pulsed laser required in lifetime testing was more powerful, and when more and deeper UCNPs were excited, the change of statistical lifetime was not obvious since the deeper UCNPs were out of the range of FRET. Fig. 4f shows the result of specificity test, and the concentration of each control group is the same of $20 \mathrm{ng} / \mathrm{mL}$. From the result, PSA quenches fluorescence intensity significantly, indicating good selectivity of this detection system.

This biochip for the detection of tumor marker is promising to be applied for home self-screening, since the upconversion emission is greatly enhanced, and the change in fluorescence under a fluorescence microscope can be clearly seen in the range of $0.1 \sim 20 \mathrm{ng} / \mathrm{mL}$.

(a)

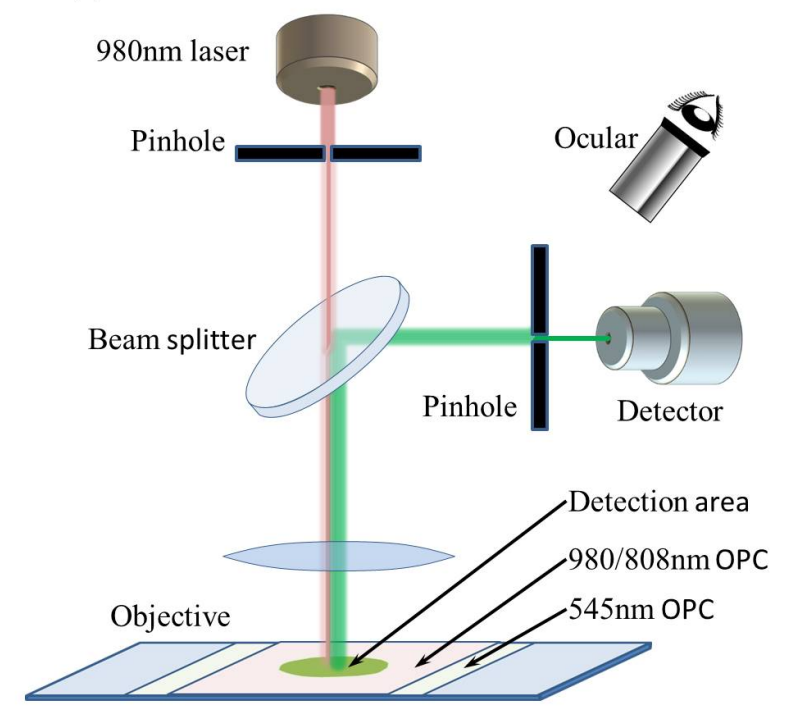

(b)

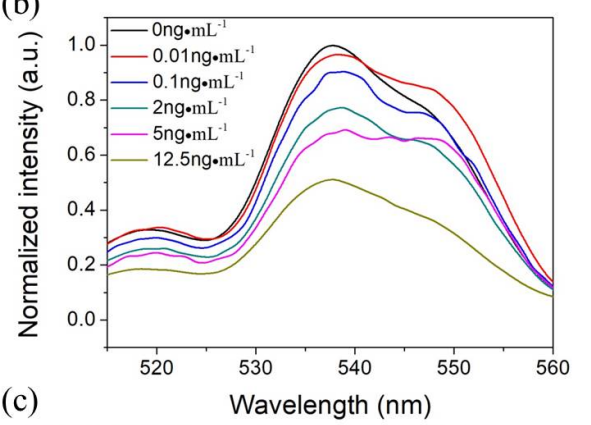

(c)

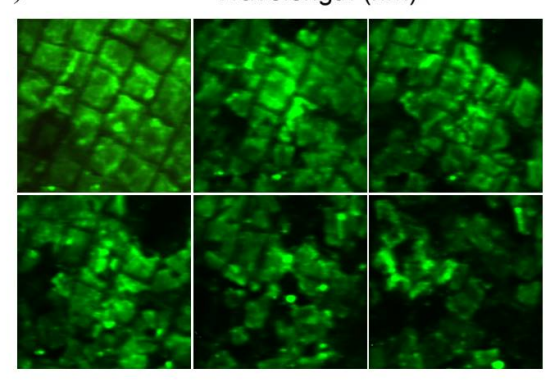

Fig. 5 (a) schematic diagram of detection process tested with confocal microscope. (b-c) emission spectra and corresponding photograph tested by confocal microscope under $980 \mathrm{~nm}$ excitation (Concentration of PSA in sample is $0,0.01,0.1,2,5,12.5 \mathrm{ng} / \mathrm{mL}$, respectively).

Fig. 5 shows the detection result tested by confocal microscope with $980 \mathrm{~nm}$ excitation. We coupled a $980 \mathrm{~nm}$ laser to a confocal microscope, and the upconversion emission can be observed through a microscope. In addition, the spectra can be tested through a microscope. Fig. 5a is a schematic diagram of the overall sensing system. In this sensing system, the results also show remarkable sensing performance with the detection limit of $0.01 \mathrm{ng} / \mathrm{mL}$, in the range of $0.1 \sim 12.5 \mathrm{ng} / \mathrm{mL}$. We made a 
comparison between our detection method and the reported methods within Table 1, which indicates the remarkable detection performance of this tumor marker sensor among the various methods. Fig. 5(b-c) shows the emission spectra and corresponding photograph tested by confocal microscope with $980 \mathrm{~nm}$ excitation. In the green fluorescent region of the photonic crystal (Fig. 5c), when the PSA is added, the brightness of the green fluorescent region is gradually decreased. Note that the color in the figure is pseudo color and visual image of the detection can be realized through the gray value. Since excessive GNPs made no difference to fluorescence quenching, in this section, the same area on the biochip was selected for the multi-detection of PSA. Enough amount of capture anti-PSA was added firstly, the amount of capture anti-PSA was calculated to be enough to meet the requirement of six experiments. After PSA and detection anti-PSA modified GNPs was subsequently added, through this sandwich detection method, PSA with different concentrations can be tested multiple times.

Table. 1 Comparison of different strategies used for PSA detection.

\begin{tabular}{lllll}
\hline Detection structure & Sensor principle & Linear range & LOD & Ref. \\
\hline MWCNT & ECL & $2.25 \sim 11.3 \mathrm{ng} / \mathrm{mL}$ & $0.88 \mathrm{ng} / \mathrm{mL}$ & {$[53]$} \\
Nanomagnetic beads & Fluorescent & $0.05 \sim 100 \mathrm{ng} / \mathrm{mL}$ & $0.05 \mathrm{ng} / \mathrm{mL}$ & {$[54]$} \\
CdTe QDs & Fluorescent & $1 \sim 40 \mathrm{ng} / \mathrm{mL}$ & $0.4 \mathrm{ng} / \mathrm{mL}$ & {$[55]$} \\
Gold NPs & SERS & $4 \sim 10 \mathrm{ng} / \mathrm{mL}$ & $0.012 \mathrm{ng} / \mathrm{mL}$ & {$[56]$} \\
MOFs & Electrochemical & $0.1 \sim 100 \mathrm{ng} / \mathrm{mL}$ & $0.06 \mathrm{ng} / \mathrm{mL}$ & {$[57]$} \\
$\begin{array}{l}\text { Mesoporous silica } \\
\text { gold film }\end{array}$ & Electrochemical & $1 \sim 300 \mathrm{ng} / \mathrm{mL}$ & $0.28 \mathrm{ng} / \mathrm{mL}$ & {$[58]$} \\
gold film & & & & \\
Gold nanodisk & SPR & $0.1 \sim 50 \mathrm{ng} / \mathrm{mL}$ & $0.091 \mathrm{ng} / \mathrm{mL}$ & {$[59]$} \\
OPC/UCNPs & FSPR & $1.7 \sim 20.4 \mathrm{ng} / \mathrm{mL}$ & $1.49 \mathrm{ng} / \mathrm{mL}$ & {$[60]$} \\
\hline
\end{tabular}

\section{Conclusion}

In summary, a novel bilayer structure OPC is designed which simultaneously matches with the excitation wavelength and emission wavelength of the UCNPs. According to the emission spectra of UCNPs on different substrates which are prepared in the same condition, this novel structure shows significantly higher 
upconversion enhancement factor than the conventional single OPC. With low excitation power density and enhanced emission intensity, the upconversion based PSA sensor was fabricated using $\mathrm{NaYF}_{4}: \mathrm{Yb}^{3+}, \mathrm{Er}^{3+} \mathrm{UCNPs}$ and $\mathrm{NaYF}_{4}: \mathrm{Yb}^{3+}$, $\mathrm{Er}^{3+} @ \mathrm{NaYF}_{4}: \mathrm{Yb}^{3+} \mathrm{UCNPs}$, respectively. Under $980 \mathrm{~nm}$ and $808 \mathrm{~nm}$ laser excitation, this detection shows a good linear relationship within the range of $0.1 \mathrm{ng} / \mathrm{mL} \sim 10$ $\mathrm{ng} / \mathrm{mL}$ and LOD of $0.01 \mathrm{ng} / \mathrm{mL}$. Since the upconversion fluorescence was greatly enhanced, this system could excited by the NIR laser with low power density of 1.7 $\mathrm{W} / \mathrm{cm}^{2}$. This enhanced upconvesion based strategy can be extended to design a universal biomaterial sensor, and applied in the areas of health assessment and disease diagnosis.

\section{Acknowledgements}

This work was supported by the National Key Research and Development Program (2016YFC0207101), the National Natural Science Foundation of China (Grant Nos. 61775080, 11874181, 61874049, 11704056) and the Key Program of NSFC-Guangdong Joint Funds of China (U1801253), Research Project of Jilin Provincial Department of Education (JJKH20200975KJ). 


\section{References}

[1] L. Wu, X. Qu, Cancer biomarker detection: recent achievements and challenges, Chemical Society Reviews, 44(2015) 2963-97.

[2] M. Wang, D.M. Wang, Q. Chen, C.X. Li, Z.Q. Li, J. Lin, Recent Advances in Glucose-Oxidase-Based Nanocomposites for Tumor Therapy, Small, 26.

[3] Z.Y. Hou, K.R. Deng, M.F. Wang, Y.H. Liu, M.Y. Chang, S.S. Huang, et al., Hydrogenated Titanium Oxide Decorated Upconversion Nanoparticles: Facile Laser Modified Synthesis and $808 \mathrm{~nm}$ Near-Infrared Light Triggered Phototherapy, Chem Mat, 31(2019) 774-84.

[4] B. Liu, C.X. Li, P.P. Yang, Z.Y. Hou, J. Lin, 808-nm-Light-Excited Lanthanide-Doped Nanoparticles: Rational Design, Luminescence Control and Theranostic Applications, Adv Mater, 29(2017) 24.

[5] D.M. Wang, B. Liu, Z.W. Quan, C.X. Li, Z.Y. Hou, B.G. Xing, et al., New advances on the marrying of UCNPs and photothermal agents for imaging-guided diagnosis and the therapy of tumors, J Mat Chem B, 5(2017) 2209-30.

[6] Y.L. Dai, H.T. Bi, X.R. Deng, C.X. Li, F. He, P.A. Ma, et al., $808 \mathrm{~nm}$ near-infrared light controlled dual-drug release and cancer therapy in vivo by upconversion mesoporous silica nanostructures, J Mat Chem B, 5(2017) 2086-95.

[7] L.Y. Zhang, Y.Y. Chen, Z.L. Li, L. Li, P. Saint-Cricq, C.X. Li, et al., Tailored Synthesis of Octopus-type Janus Nanoparticles for Synergistic Actively-Targeted and Chemo-Photothermal Therapy, Angew Chem-Int Edit, 55(2016) 2118-21.

[8] F. Bray, J. Ferlay, I. Soerjomataram, R.L. Siegel, L.A. Torre, A. Jemal, Global cancer statistics 2018: GLOBOCAN estimates of incidence and mortality worldwide for 36 cancers in 185 countries, Ca-a Cancer Journal for Clinicians, 68(2018) 394-424.

[9] J. Wu, Z.F. Fu, F. Yan, H.X. Ju, Biomedical and clinical applications of immunoassays and immunosensors for tumor markers, Trac-Trends In Analytical Chemistry, 26(2007) 679-88.

[10] M. Perfezou, A. Turner, A. Merkoci, Cancer detection using nanoparticle-based sensors, Chemical Society Reviews, 41(2012) 2606-22.

[11] G.F. Zheng, F. Patolsky, Y. Cui, W.U. Wang, C.M. Lieber, Multiplexed electrical detection of cancer markers with nanowire sensor arrays, Nat Biotechnol, 23(2005) 1294-301.

[12] G.J. Zhang, Z.G. Liu, L.F. Fan, Y.J. Guo, Electrochemical prostate specific antigen aptasensor based on hemin functionalized graphene-conjugated palladium nanocomposites, Microchim Acta, 185(2018) 8.

[13] P. Das, A. Sedighi, U.J. Krull, Cancer biomarker determination by resonance energy transfer using functional fluorescent nanoprobes, Anal Chim Acta, 1041(2018) 1-24.

[14] M.M. Shang, C.X. Li, J. Lin, How to produce white light in a single-phase host?, Chemical Society Reviews, 43(2014) 1372-86.

[15] W.C.W. Chan, D.J. Maxwell, X.H. Gao, R.E. Bailey, M.Y. Han, S.M. Nie, Luminescent quantum dots for multiplexed biological detection and imaging, Curr Opin Biotechnol, 13(2002) 40-6.

[16] I.L. Medintz, H. Mattoussi, Quantum dot-based resonance energy transfer and its growing application in biology, Phys Chem Chem Phys, 11(2009) 17-45.

[17] A.M. Smith, S.M. Nie, Semiconductor Nanocrystals: Structure, Properties, and Band Gap Engineering, Accounts Chem Res, 43(2010) 190-200.

[18] M.O. Noor, U.J. Krull, Paper-Based Solid-Phase Multiplexed Nucleic Acid Hybridization Assay with Tunable Dynamic Range Using Immobilized Quantum Dots As Donors in Fluorescence Resonance Energy Transfer, Anal Chem, 85(2013) 7502-11. 
[19] X.Y. Huang, S.Y. Han, W. Huang, X.G. Liu, Enhancing solar cell efficiency: the search for luminescent materials as spectral converters, Chemical Society Reviews, 42(2013) 173-201.

[20] M.B. de la Mora, O. Amelines-Sarria, B.M. Monroy, C.D. Hernandez-Perez, J.E. Lugo, Materials for downconversion in solar cells: Perspectives and challenges, Sol Energy Mater Sol Cells, 165(2017) 59-71.

[21] J.C. Goldschmidt, S. Fischer, Upconversion for Photovoltaics - A Review of Materials, Devices and Concepts for Performance Enhancement (vol 3, pg 510, 2015), Adv Opt Mater, 3(2015) 1487-.

[22] J.H. Zhang, Z.D. Hao, J. Li, X. Zhang, Y.S. Luo, G.H. Pan, Observation of efficient population of the red-emitting state from the green state by non-multiphonon relaxation in the Er3+-Yb3+ system, Light-Science \& Applications, 4(2015) 6.

[23] W.P. Qin, Z.Y. Liu, C.N. Sin, C.F. Wu, G.S. Qin, Z. Chen, et al., Multi-ion cooperative processes in Yb3+ clusters, Light-Science \& Applications, 3(2014) 6.

[24] Z.Y. Hou, K.R. Deng, C.X. Li, X.R. Deng, H.Z. Lian, Z.Y. Cheng, et al., 808 nm Light-triggered and hyaluronic acid-targeted dual-photosensitizers nanoplatform by fully utilizing $\mathrm{Nd} 3(+)$-sensitized upconversion emission with enhanced anti-tumor efficacy, Biomaterials, 101(2016) 32-46.

[25] D.M. Yang, P.A. Ma, Z.Y. Hou, Z.Y. Cheng, C.X. Li, J. Lin, Current advances in lanthanide ion $(\operatorname{Ln}(3+))$-based upconversion nanomaterials for drug delivery, Chemical Society Reviews, 44(2015) 1416-48.

[26] Z.Y. Hou, Y.X. Zhang, K.R. Deng, Y.Y. Chen, X.J. Li, X.R. Deng, et al., UV-Emitting Upconversion-Based TiO2 Photosensitizing Nanoplatform: Near-Infrared Light Mediated in Vivo Photodynamic Therapy via Mitochondria-Involved Apoptosis Pathway, ACS Nano, 9(2015) 2584-99.

[27] F. Wang, S.H. Wen, H. He, B.M. Wang, Z.G. Zhou, O. Shimoni, et al., Microscopic inspection and tracking of single upconversion nanoparticles in living cells, Light-Science \& Applications, 7(2018) 6.

[28] B. Liu, Y.Y. Chen, C.X. Li, F. He, Z.Y. Hou, S.S. Huang, et al., Poly(Acrylic Acid) Modification of Nd3+-Sensitized Upconversion Nanophosphors for Highly Efficient UCL Imaging and pH-Responsive Drug Delivery, Adv Funct Mater, 25(2015) 4717-29.

[29] C.X. Li, D.M. Yang, P.A. Ma, Y.Y. Chen, Y. Wu, Z.Y. Hou, et al., Multifunctional Upconversion Mesoporous Silica Nanostructures for Dual Modal Imaging and In Vivo Drug Delivery, Small, 9(2013) 4150-9.

[30] D.M. Yang, X.J. Kang, P.A. Ma, Y.L. Dai, Z.Y. Hou, Z.Y. Cheng, et al., Hollow structured upconversion luminescent NaYF4:Yb3+, Er3+ nanospheres for cell imaging and targeted anti-cancer drug delivery, Biomaterials, 34(2013) 1601-12.

[31] Z.Y. Hou, C.X. Li, P.A. Ma, G.G. Li, Z.Y. Cheng, C. Peng, et al., Electrospinning Preparation and Drug-Delivery Properties of an Up-conversion Luminescent Porous NaYF4:Yb3+, Er3+@Silica Fiber Nanocomposite, Adv Funct Mater, 21(2011) 2356-65.

[32] R.R. Deng, F. Qin, R.F. Chen, W. Huang, M.H. Hong, X.G. Liu, Temporal full-colour tuning through non-steady-state upconversion, Nat Nanotechnol, 10(2015) 237-42.

[33] F. Wang, R.R. Deng, J. Wang, Q.X. Wang, Y. Han, H.M. Zhu, et al., Tuning upconversion through energy migration in core-shell nanoparticles, Nat Mater, 10(2011) 968-73.

[34] J. Wang, R.R. Deng, M.A. MacDonald, B.L. Chen, J.K. Yuan, F. Wang, et al., Enhancing multiphoton upconversion through energy clustering at sublattice level, Nat Mater, 13(2014) 157-62.

[35] C.X. Li, J. Lin, Rare earth fluoride nano-/microcrystals: synthesis, surface modification and application, J Mater Chem, 20(2010) 6831-47.

[36] C.X. Li, Z.Y. Hou, C.M. Zhang, P.P. Yang, G.G. Li, Z.H. Xu, et al., Controlled Synthesis of $\operatorname{Ln}(3+)(\operatorname{Ln}=$ 
Tb, Eu, Dy) and V5+ lon-Doped YPO4 Nano-/Microstructures with Tunable Luminescent Colors, Chem Mat, 21(2009) 4598-607.

[37] X. Chen, Y.S. Zhu, D.L. Zhou, W. Xu, J.Y. Zhu, G.C. Pan, et al., Size-dependent downconversion near-infrared emission of NaYF4:Yb3+,Er3+ nanoparticles, J Mater Chem C, 5(2017) 2451-8.

[38] Z. Li, Q. Sun, Y. Zhu, B. Tan, Z.P. Xu, S.X. Dou, Ultra-small fluorescent inorganic nanoparticles for bioimaging, J Mat Chem B, 2(2014) 2793-818.

[39] C.Y. Liu, Z.Y. Gao, J.F. Zeng, Y. Hou, F. Fang, Y.L. Li, et al., Magnetic/Upconversion Fluorescent NaGdF4:Yb,Er Nanoparticle-Based Dual-Modal Molecular Probes for Imaging Tiny Tumors in Vivo, ACS Nano, 7(2013) 7227-40.

[40] N. Liu, W.P. Qin, G.S. Qin, T. Jiang, D. Zhao, Highly plasmon-enhanced upconversion emissions from Au@beta-NaYF4:Yb,Tm hybrid nanostructures, Chem Commun, 47(2011) 7671-3.

[41] J.J. He, W. Zheng, F.L. Ligmajer, C.F. Chan, Z.Y. Bao, K.L. Wong, et al., Plasmonic enhancement and polarization dependence of nonlinear upconversion emissions from single gold nanorod@SiO2@CaF2:Yb3+,Er3+ hybrid core-shell-satellite nanostructures, Light-Science \& Applications, 6(2017) 11.

[42] Q.C. Sun, H. Mundoor, J.C. Ribot, V. Singh, Smalyukh, II, P. Nagpal, Plasmon-Enhanced Energy Transfer for Improved Upconversion of Infrared Radiation in Doped-Lanthanide Nanocrystals, Nano Lett, 14(2014) 101-6.

[43] S. Noda, M. Fujita, T. Asano, Spontaneous-emission control by photonic crystals and nanocavities, Nat Photonics, 1(2007) 449-58.

[44] Z. Yin, Y.S. Zhu, W. Xu, J. Wang, S. Xu, B. Dong, et al., Remarkable enhancement of upconversion fluorescence and confocal imaging of PMMA Opal/NaYF4:Yb3+, Tm3+/Er3+ nanocrystals, Chem Commun, 49(2013) 3781-3.

[45] B.Y. Ding, C. Hrelescu, N. Arnold, G. Isic, T.A. Klar, Spectral and Directional Reshaping of Fluorescence in Large Area Self-Assembled Plasmonic-Photonic Crystals, Nano Lett, 13(2013) 378-86.

[46] P.W. Zhou, D.L. Zhou, L. Tao, Y.S. Zhu, W. Xu, S. Xu, et al., 320-fold luminescence enhancement of $\mathrm{Ru}(\mathrm{dpp})(3) \mathrm{Cl}-2$ dispersed on PMMA opal photonic crystals and highly improved oxygen sensing performance, Light-Science \& Applications, 3(2014) 9.

[47] D.L. Zhou, P.W. Zhou, D.L. Liu, W. Xu, Y.S. Zhu, S. Xu, et al., Modulation of upconversion white light emission in PMMA/NaYF4:Yb3+, Er3+, Tm3+ composite photonic crystals, Opt Lett, 39(2014) 4619-22.

[48] Z. Yin, H. Li, W. Xu, S.B. Cui, D.L. Zhou, X. Chen, et al., Local Field Modulation Induced Three-Order Upconversion Enhancement: Combining Surface Plasmon Effect and Photonic Crystal Effect, Adv Mater, 28(2016) 2518-+.

[49] H.Q. Chen, F. Yuan, S.Z. Wang, J. Xu, Y.Y. Zhang, L. Wang, Aptamer-based sensing for thrombin in red region via fluorescence resonant energy transfer between NaYF4:Yb,Er upconversion nanoparticles and gold nanorods, Biosensors \& Bioelectronics, 48(2013) 19-25.

[50] Q. Long, H.T. Li, Y.Y. Zhang, S.Z. Yao, Upconversion nanoparticle-based fluorescence resonance energy transfer assay for organophosphorus pesticides, Biosensors \& Bioelectronics, 68(2015) 168-74. [51] J.H. Peng, Y.H. Wang, J.L. Wang, X. Zhou, Z.H. Liu, A new biosensor for glucose determination in serum based on up-converting fluorescence resonance energy transfer, Biosensors \& Bioelectronics, 28(2011) 414-20.

[52] W. Xu, Y.S. Zhu, X. Chen, J. Wang, L. Tao, S. Xu, et al., A novel strategy for improving upconversion luminescence of NaYF4:Yb, Er nanocrystals by coupling with hybrids of silver plasmon nanostructures and poly(methyl methacrylate) photonic crystals, Nano Res, 6(2013) 795-807. 
[53] A. Juzgado, A. Solda, A. Ostric, A. Criado, G. Valenti, S. Rapino, et al., Highly sensitive electrochemiluminescence detection of a prostate cancer biomarker, J Mat Chem B, 5(2017) 6681-7.

[54] B.X. Li, J. Liu, H. Zhou, Amplified fluorescence detection of serum prostate specific antigen based on metal-dependent DNAzyme assistant nanomachine, Anal Chim Acta, 1008(2018) 96-102.

[55] Y. Chen, X.Y. Guo, W. Liu, L. Zhang, Paper-based fluorometric immunodevice with quantum-dot labeled antibodies for simultaneous detection of carcinoembryonic antigen and prostate specific antigen, Microchim Acta, 186(2019) 9.

[56] Z. Cheng, N. Choi, R. Wang, S. Lee, K.C. Moon, S.Y. Yoon, et al., Simultaneous Detection of Dual Prostate Specific Antigens Using Surface-Enhanced Raman Scattering-Based Immunoassay for Accurate Diagnosis of Prostate Cancer, ACS Nano, 11(2017) 4926-33.

[57] S.K. Bhardwaj, A.L. Sharma, N. Bhardwaj, M. Kukkar, A.A.S. Gill, K.H. Kim, et al., TCNQ-doped Cu-metal organic framework as a novel conductometric immunosensing platform for the quantification of prostate cancer antigen, Sens Actuator B-Chem, 240(2017) 10-7.

[58] W. Argoubi, A. Sanchez, C. Parrado, N. Raouafi, R. Villalonga, Label-free electrochemical aptasensing platform based on mesoporous silica thin film for the detection of prostate specific antigen, Sens Actuator B-Chem, 255(2018) 309-15.

[59] G. Erturk, H. Ozen, M.A. Tumer, B. Mattiasson, A. Denizli, Microcontact imprinting based surface plasmon resonance (SPR) biosensor for real-time and ultrasensitive detection of prostate specific antigen (PSA) from clinical samples, Sens Actuator B-Chem, 224(2016) 823-32.

[60] Y. Khan, A.R. Li, L. Chang, L.D. Li, L. Guo, Gold nano disks arrays for localized surface plasmon resonance based detection of PSA cancer marker, Sens Actuator B-Chem, 255(2018) 1298-307. 